\title{
Réélaboration et structure : l'héritage dans la langue et en linguistique
}

\section{Pierre Cotte}

\section{(2) OpenEdition}

\section{Journals}

Édition électronique

URL : http://journals.openedition.org/asp/2296

DOI : 10.4000/asp.2296

ISBN : 978-2-8218-0380-0

ISSN : 2108-6354

Éditeur

Groupe d'étude et de recherche en anglais de spécialité

Édition imprimée

Date de publication : 1 décembre 1999

Pagination : 7-27

ISSN : 1246-8185

\section{Référence électronique}

Pierre Cotte, "Réélaboration et structure : l'héritage dans la langue et en linguistique », ASp [En ligne], 23-26 | 1999, mis en ligne le 05 mars 2011, consulté le 02 mai 2019. URL : http://

journals.openedition.org/asp/2296; DOI : 10.4000/asp.2296

Ce document a été généré automatiquement le 2 mai 2019.

Tous droits réservés 


\title{
Réélaboration et structure : l'héritage dans la langue et en linguistique
}

\author{
Pierre Cotte
}

1 La logique classique définit le genre et l'espèce en termes d'extension et d'inclusion: si une classe $A$ est une partie de l'extension d'une classe $B$, $A$ est dit espèce de $B$ et $B$ genre de $\mathrm{A}^{1}$. L'extension étant fonction de l'intension du concept désignant la classe - de l'ensemble des propriétés pertinentes synthétisées par celui-ci - la définition signifie que toutes les propriétés du genre s'observent aussi dans l'espèce, sinon la seconde ne serait pas une partie du premier, sans que l'inverse soit vrai : pour être distinguée, l'espèce doit avoir des traits propres ignorés du genre (l'espèce saumon a tous les traits du genre poisson, et certains que le second n'a pas). Une théorie génétique tire aussi bien l'espèce du genre que le genre de l'espèce, mais les implications sont différentes. Si le genre est premier, l'espèce se constitue par ajout de traits : elle garde la mémoire du genre et la perpétue. Si l'espèce est première, le genre s'obtient par soustraction de traits; il oublie l'espèce, rompt avec elle. D'un côté, la continuité, les liens positifs, l'augmentation; de l'autre, la coupure et la diminution. Loi cognitive ou conditionnement culturel, l'esprit donne souvent la préférence aux rapports positifs unifiants. Dans son analyse des hiérarchies de type espèce de, l'intelligence artificielle dit que l'espèce « hérite » du genre ${ }^{2}$ ; pour elle, le genre précède les espèces, les engendre, subsiste en elles; les espèces naissent du genre, le conservent, en ajoutant leurs traits.

2 En linguistique, l'intuition d'un accroissement du sens est au cœur de la théorie de la «psychosystématique du langage ». Pour Gustave Guillaume, son inventeur, le temps, substrat obligé de l'opération de la pensée, construit le sens de façon continue et cumulative. Son cinétisme, intercepté précocement, livre une notion sémantiquement légère, l'équivalent du genre ci-dessus; intercepté tardivement, il donne des notions 
enrichies, comme peuvent l'être celles désignant des espèces. Le dynamisme constructeur étant continu, les notions tardives sont les avatars des précoces, dont elles "héritent " également et le système est positif. Les unités ne sont pas seulement « ce que les autres ne sont pas ", comme dans un système saussurien hanté par le négatif. Elles sont d'abord ce que les autres ont été ; elles y réfèrent, en font la sommation : les identités sous-tendent les différences ${ }^{3}$. Les principes à l'œuvre dans l'« idéogénèse », l'opération créatrice de la signification des lexèmes, informent aussi les systèmes grammaticaux. Le temps opératif porte l'axe sur lequel s'échelonnent selon un degré croissant de définition le mode nominal du verbe, le subjonctif et l'indicatif ${ }^{4}$. Il anime aussi le système de l'article, qui prend la forme originale d'un «tenseur binaire».

3 Constatant que chaque article accepte un référent générique ou spécifique, G. Guillaume imagine que ce système se compose de deux tensions successives orientées de l'universel au singulier (indéfini) puis du singulier à l'universel (défini). Chaque tension allie ces extrêmes dans des proportions variables selon le point d'interception; la spécificité des articles tient à l'ordre des tensions sur l'axe temporel. À l'entrée du système, l'indéfini conduit du plan puissanciel de la langue, où le nom est pur potentiel de référence, au discours effectif où s'exprime, ici et maintenant, une référence particulière générique ou spécifique ; typiquement, il introduit un référent dans le texte. Une interception précoce donne un générique, orienté cependant par le cinétisme vers le spécifique : le référent semble sous nos yeux (A car has four wheels); on en a l'expérience (A dog is faithful). Les interceptions suivantes trouvent une unité toujours mieux singularisée quantitativement et qualitativement (I saw a funny dog). Second dans le système, le défini commence avec le discours effectif, dont il continue les références et il retourne à la langue. En interception précoce il reste proche du pôle singulier où l'indéfini s'arrête et sa référence est spécifique. Typiquement, il pointe dans le texte réalisé et reprend une référence que l'apport du contexte rend désormais unique en son genre (The dog I saw). En interception tardive, le cinétisme l'éloignant du spécifique, il tend vers le générique. Plus d'échantillon, d'expérience, ni d'anaphore étroite. Cependant, à la sortie du système de l'article, le référent a intégré les discours tenus sur lui. Ses qualités structurelles sont désormais des stéréotypes à valeur distinctive (The dog is faithful). Grâce aux textes passés, il possède des qualités contingentes non prévues par la seule notion nominale, que la saisie précoce d'un indéfini interdit d'exprimer (The car has revolutionized modern life /*A car has revolutionized modern life). Le sens se construit; sa mémoire se conserve: le discours a enrichi le nom qui retourne à la langue ${ }^{5}$.

\section{2}

4 Les travaux de G. Guillaume ont inspiré de nombreux linguistes ${ }^{6}$. La " théorie des phases » de H. Adamczewski (1982), reprenant l'idée du temps opératif, propose du tenseur binaire une version allégée où priment mémoire du sens et anaphore. L'axe orienté du temps est divisé en deux parties. À la différence des parties du tenseur binaire guillaumien, celles-ci ne joignent pas des pôles cognitifs et aucune interception précoce ou tardive n'y génère d'effet de sens. Elles se contentent d'accueillir des formes grammaticales synonymes, qu'elles ordonnent et enchaînent. La seconde des formes mises en système présuppose la première ; elle en constitue une variante élaborée, en lui ajoutant un commentaire modal ou en favorisant des opérations énonciatives complexes. 
5 Une illustration célèbre de la théorie est l'analyse des "temps simples » et de la périphrase be + ing en anglais. Pour $\mathrm{H}$. Adamczewski, les verbes lexicaux conjugués au présent ou au prétérit correspondent à une phase énonciative première dite "rhématique ", où l'énoncé introduit et identifie simplement un procès. La périphrase s'emploie lors d'une phase anaphorique seconde, "thématique ", où la référence précédemment construite est reprise. Elle accompagne diverses modalisations, subordinations, mises en relation dans le texte. À la suite de G. Guillaume, H. Adamczewski considère les formes linguistiques comme les traces d'un processus sémantique. La complexité formelle supérieure de la périphrase, laisse-t-il entendre, traduit la complexité énonciative de la deuxième phase et les morphèmes ing et be reflètent deux étapes du sens (1982: 56-76). L'opération impliquée par ing est une nominalisation. "Présupposante » et " anaphorique ", celle-ci reprend une proposition assertée conjuguée simplement, fait disparaître le sujet et transforme le prédicat en un syntagme nominal intégrable à une autre structure pouvant exprimer une modalité (ex. : I heard the girl I loved insulted $\rightarrow$ hearing the girl I loved insulted $\rightarrow$ I couldn't stand hearing the girl I loved insulted). L'association de ce syntagme au verbe « existentiel » be produit la périphrase.

6 Quand celle-ci est conjuguée, be lie le sujet syntaxique et le syntagme nominal issu de l'ancien prédicat. Même si ce dernier contient un objet, l'énoncé, constitué de deux grands blocs seulement, est « binaire ». Le lien entre le sujet et le prédicat est orienté : be rapporte le second au premier et l'énoncé est implicitement attributif. Le référent du sujet n'est pas considéré pour son rôle dans le procès, par exemple comme agent ; c'est un support d'incidence et il est statique: le seul agent véritable, dit Adamczewski, est l'énonciateur opérant sur l'énoncé présupposé. L'anglais valorisant la relation du prédicat au sujet, la périphrase rétablirait celle-ci quand le procès - activité ou action - regarde normalement vers le monde ou vers l'objet; les processus mentaux ou les perceptions, naturellement orientés vers le sujet, la demandent moins.

$7 \quad$ Attribuant le prédicat nominalisé au sujet, be matérialise la relation prédicative et en fait une cible pour diverses opérations qui «déclenchent " la périphrase : verbes recteurs comme know, think, hear, see, realize, adverbes, négation, etc. Dans He was seeing a real war for the first time, la cible du circonstant n'est donc pas see mais be ; la glose proposée est "C'était la première fois qu'il y avait relation ». L'idée de voir une guerre ayant été mentionnée ou étant implicite, l'énoncé établit seulement une relation avec un sujet particulier et le circonstant commente cette relation.

8 Ici, la théorie est à des lieues du discours habituel, qui parle de «forme progressive », d'action " inachevée ", " en cours », de procès "dynamique ", "duratif », ou encore de coïncidence entre le procès et un instant repère. Adamczewski ne cherche pas à concilier les points de vue: il traite de haut l'approche aspectuelle classique, avec quelques critiques rapides. $1^{\circ}$ ) L'équivalence souvent évoquée entre be + ing et être en train de est partielle (You are forgetting your lighter ne se traduit pas * Tu es en train d'oublier ton briquet). $2^{\circ}$ ) De même celle entre was + ing et l'imparfait (Le soleil brillait peut être The sun shone autant que The sun was shining). $3^{\circ}$ ) L'opposition ponctuel/duratif ne va pas de soi. Comment doit-on qualifier Elle régna vingt ans ? $4^{\circ}$ ) La durée est sans pertinence dans de nombreux énoncés en be + ing (ex: He was seeing a war for the first time). $5^{\circ}$ ) La mention de la durée par un circonstant n'impose pas la périphrase (ex : Hercule Poirot looked a long time at the red agitated face of Superintendent Spence). Il conclut que be + ing ne reflète pas la 
situation extralinguistique, mais indique une anaphore, contextuelle ou situationnelle, et une opération sur l'énoncé.

\section{3}

9 La critique est trop rapide et trop caricaturale parfois pour convaincre. $1^{\circ}$ ) Certes, l'équivalence entre l'imparfait ou être en train de et les formes pertinentes de be + ing est partielle, mais cela interdit-il que la différence puisse être aspectuelle? Pour l'auteur, l'aspect est dans l'extralinguistique; il est objectif, simple et banal, alors que be + ing participe de l'énonciation, est subjectif et complexe. Mais les nombreuses études sur les formes françaises concernées montrent leur richesse et proposent des théories où l'aspect s'avère une construction énonciative élaborée, soumise à de multiples contraintes et répondant souvent à des choix narratifs et stylistiques ${ }^{7}$. Rien n'empêche donc be + ing, qui a ces qualités, d'être aspectuel malgré tout.

$102^{\circ}$ ) L'opposition ponctuel/duratif peut paraître fragile. Elle ne repose sur rien de précis; les effets de sens sont cependant bien réels : dans l'énoncé En 1860 elle régnait depuis dix ans , le règne paraît extensible; dans Elle régna pendant vingt ans, sa borne finale identifiée semble le contracter même si la durée est supérieure. Le linguiste doit s'occuper des faits récurrents sur lesquels les locuteurs s'accordent : s'il élabore une théorie non-aspectuelle des marques, il doit veiller qu'elle explique aussi les effets aspectuels. Peut-être les opérations énonciatives à l'origine de be + ing rendent-elles compte de l'effet progressif. $\mathrm{Au}$ lieu de lui refuser toute importance, Adamczewski aurait dû proposer une théorie plus intégrante le prévoyant.

$113^{\circ}$ ) Des théories aspectuelles de be + ing Adamczewski retient la dimension de l'accomplissement, mais il néglige la concomitance, théorisée par les énonciativistes. Cet oubli est gênant. On admettra que le caractère inaccompli et progressif du procès n'est pas au premier plan dans He was seeing a real war for the first time ou When a girl of twenty marries a man close to eighty, it is obvious that she is marrying him for money. La concomitance reste : dans ces énoncés, il est conforme à l'intuition de dire que be + ing fait coïncider le procès avec un repère temporel précis (He was seeing a real war then, She is marrying him for money then). Or ce repère temporel est précis parce qu'il est aussi le repère d'un premier procès (un incident narré encore présent à l'esprit, marry a man close to eighty respectivement), si bien qu'il n'y a qu'un pas de la coïncidence temporelle à la coïncidence textuelle de l'anaphore. La théorie classique dira que la marque be + ing fait coïncider le procès qu'elle détermine avec un repère temporel puis avec un autre procès lié à ce repère. Une théorie de l'anaphore dira qu'elle le fait coïncider avec un premier procès et secondairement avec sa temporalité. Quoi qu'on dise, la langue est complexe; le linguiste doit le reconnaître.

$\left.124^{\circ}\right)$ Contre la théorie aspectuelle, il est affirmé parfois que les locuteurs employant be + ing ne « veulent pas dire » que le procès est en cours. De fait, on emploie une locution comme be in progress pour communiquer cette idée spécifiquement, et on construit normalement $H e$ 's watching television ou He's having dinner non pour signifier principalement que le procès mentionné a telle propriété aspectuelle mais pour indiquer, par exemple, quelle est l'activité actuelle du sujet ou pourquoi il ne doit pas être dérangé. L'observation est judicieuse et on aimerait mieux connaître la fonction pragmatique des énoncés ayant telle ou telle marque grammaticale. Cependant, elle ne constitue pas un argument très 
fort : les théories classiques de be + ing ne prétendent pas que la valeur aspectuelle est la principale visée d'un énoncé, mais seulement qu'elle contribue à sa cohérence en articulant le procès à une situation repère ou à un autre procès du texte.

$\left.5^{\circ}\right)$ O. Jespersen pensait que la périphrase be + ing indique moins la durée qu'elle ne fait du procès un cadre temporel où est situé, par exemple, l'instant d'énonciation : He is writing (now), ou un autre procès : He was writing when I entered (1909-49, IV : $178 \mathrm{sq}$ ). La théorie traduit fidèlement notre intuition de nombreux énoncés mais elle échoue avec When a girl of twenty marries a man close to eighty, it is obvious that she is marrying for money, où aucune inclusion stricto sensu n'est signifiée. Parce que le procès déterminé par be + ing n'est pas toujours un cadre, Adamczewski rejetterait la théorie de Jespersen. Pour ce dernier et pour de nombreux grammairiens traditionnels, be + ing révèle sa nature dans un emploi estimé représentatif. Pour Adamczewski, qu'influence le structuralisme européen, la bonne analyse se détourne des emplois, même fréquents, jugés superficiels, et débusque un trait caché actualisé à chaque occurrence, où est l'essence de la forme. Dans cette perspective, l'« invariant » de be + ing n'est pas un trait sémantico-référentiel observable en discours, mais un mécanisme de construction du sens, une opération abstraite mettant en forme la matière linguistique. C'est, dans la genèse de la périphrase, ce qu'on peut appeler l'« anaphore de construction", qui reprend pour le nominaliser un premier énoncé (cf. supra). Adamczewski pense que la mise au jour de cette anaphore, qui serait l'identité secrète de be + ing, clôt la recherche. On ne peut pas le suivre pour deux grandes raisons : a) dans sa grammaire, l'opposition de phases et l'anaphore sont sollicitées, peutêtre à juste titre, pour distinguer un tel nombre de marqueurs qu'elles ne peuvent constituer l'identité d'aucun. D'autres périphrases verbales nominalisent vraisemblablement une première proposition; dès lors, comment be + ing se résumerait-il à l'anaphore de construction ? Peut-être son invariant n'est-il pas un mécanisme unique mais plusieurs mécanismes enchaînés et prend-il en compte finalement l'aspect, dont on ne se débarrasse pas si vite. b) en première analyse, un invariant aussi général que celui proposé ne devrait favoriser aucune valeur aspectuelle particulière et la périphrase devrait avoir dans ce domaine les emplois les plus variés. Or, ce n'est pas le cas. i) Les effets de sens de celle-ci sont limités et spécialisés : concomitance soulignée, inclusion par le procès cadre d'un repère ou d'un autre procès et visée sécante (cf. supra) ii) Elle est la seule construction verbale grammaticalisée à les exprimer. Cette spécialisation suppose une disposition, peut-être une propension, que la théorie devrait expliquer, éventuellement par l'invariant abstrait. Elle le ferait si elle était guillaumienne: en psychosystématique, la langue prépare et conditionne le discours en dotant les mots grammaticaux d'un «signifié de puissance » de même nature sémantique que les effets. Faute de le faire, la théorie discutée apparaît incomplète : un comportement fréquent doit être considéré pertinent pour la caractérisation d'une forme.

$6^{\circ}$ ) Pour Adamczewski, la rareté de la périphrase quand la durée du procès est évoquée (cf. supra, Hercule Poirot looked for a long time at the red agitated face...) serait un dernier argument contre la valeur aspectuelle. L'argument, toutefois, suppose réunies deux conditions : a) qu'une seule sorte de durée existe ; b) que l'information d'un circonstant doive toujours être relayée, dans un énoncé, par une détermination verbale. Si cette contrainte était avérée, une association régulière entre be + ing et un circonstant de durée signifierait que le premier est "duratif»; une association rare devrait signifier au contraire, comme veut Adamczewski, que be + ing est sans rapport avec l'aspect. Ces conditions ne sont pas remplies. a') une distinction minimale doit être faite entre une 
durée mesurée et non mesurée. La première demande à être délimitée ; la seconde, non. Peut-être la périphrase est-elle plus difficile avec la durée mesurée parce qu'elle exprime, comme veut la tradition, du "progressif». b') la contrainte imaginée en b) n'est nullement démontrée. La langue pratique la redondance mais aussi l'économie et on pourrait imaginer qu'une loi inverse interdise au verbe de répéter ce que le circonstant a déjà dit; dans cette hypothèse, la rareté de be + ing dans le contexte de for a long time pourrait être l'indice d'une valeur durative de la périphrase!

Vraisemblablement aucune contrainte absolue n'existe et les règles décidant des combinaisons de marqueurs restent inconnues : refusons-nous une association parce que les significations un instant rapprochées sont incompatibles ou parce que l'une contient déjà l'autre? Faute de poser clairement cette question essentielle pour une syntaxe sémantique, les auteurs favorisent aléatoirement la contradiction ou l'identité : plausibles individuellement, leurs solutions sont incohérentes prises ensemble. En attendant la réponse, l'argument présenté se révèle fragile. Il n'est pas démontré que be + ing est sans rapport avec l'aspect et la théorie d'Adamczewski réduit abusivement la périphrase à un mécanisme de construction. Pour autant, l'intuition de l'anaphore nominalisante me paraît juste ; j'en propose une version enrichie.

\section{4}

Faisant l'hypothèse que le sens se construit dans le temps et que la forme enregistre certaines de ses étapes secrètes, le projet guillaumien porte en germe une syntaxe originale, sémantique et dynamique, qui identifierait les niveaux de la hiérarchie des constituants et les moments de l'élaboration du sens. G. Guillaume ne l'inventa pas car il s'intéressait surtout, comme les linguistes européens de son temps, au rapport entre le sens et les signes minimaux (mots ou parties de mots). Plus tard, dans un autre contexte, la grammaire générative refusa d'envisager entre le sens et la syntaxe un rapport organique; cependant, dans sa version transformationnelle elle introduisit implicitement le temps en tirant les structures linguistiques visibles de structures "profondes" invisibles par une série d'opérations rigoureusement ordonnées. En un sens, Adamczewski fait une synthèse des démarches quand il suggère qu'un énoncé en be + ing est une phrase complexe où une subordonnée nominalisant une première proposition invisible est dominée par une seconde qui la modalise ou la lie à une autre partie du texte. Pour cet auteur cependant, une nouvelle élaboration garde en mémoire la précédente sans plus. Je fais l'hypothèse qu'elle l'analyse également et que la structure se complexifie en s'explicitant. L'idée de « réélaboration » exprime cette intuition.

\section{5}

On abordera la question par la proximité du temps et de l'aspect. Perçue des grammairiens traditionnels qui appellent «présent » et "passé » les participes du verbe, bien connue des historiens de la langue qui observent souvent des systèmes temporels naître de l'aspect, elle est négligée des spécialistes contemporains de linguistique synchronique. Pour les structuralistes, par exemple, l'emploi par la majorité des verbes d'une même forme au prétérit et au participe passé en anglais contemporain est sans signification positive. Ce fait indiquerait seulement une "pauvreté morphologique »; il occulterait la distinction syntaxique essentielle entre formes conjuguée et non conjuguée 
du verbe et il demanderait à être corrigé par une symbolisation explicite $\operatorname{Ved}_{1} / \mathrm{Ved}_{2}$ ou $\mathrm{Ved} / \mathrm{Ven}$. Juger la distinction gave/given supérieure à l'identité lived/lived au point d'effacer toute trace de la seconde, c'est opposer la langue à elle-même et construire une représentation idéalisée peut-être fallacieuse. À cette attitude finalement normative qui juge un peu vite de ce qui est pertinent, on préférera une théorie ouverte et curieuse donnant au plus grand nombre d'observables leur juste place dans un système complexe.

On comprend la qualification traditionnelle des participes. Dans son emploi temporel, le prétérit, ou tout autre temps du passé, signifie l'antériorité à l'instant repère de l'énonciation. Le participe passé marque l'accompli ; il signifie que le procès a atteint une deuxième borne et il situe celui-ci implicitement dans l'avant d'un repère d'où la totalité réalisée peut être saisie rétrospectivement. Le présent signifie la simultanéité du procès ou d'un de ses instants avec l'instant d'énonciation. Typiquement, le participe présent montre le procès en accomplissement, tel qu'il apparait quand il coïncide avec l'instant d'observation. Ces similitudes ont motivé la dénomination traditionnelle des participes. Elles laissent entendre que les formes diffèrent sur un fond d'identité et qu'une genèse, comme il a été suggéré en 1., en construit de nouvelles dans le domaine de plus anciennes. Souvent, la catégorie du temps développe et précise celle de l'aspect; dans l'histoire de l'anglais, les périphrases à valeur aspectuelle semblent au contraire issues des temps. Les participes perpétuent ces derniers en les analysant: du rapport à l'énonciation, ils retiennent abstraitement un rapport à un repère quelconque - par là, ils réélaborent; les périphrases auxquelles ils contribuent continuent et réélaborent aussi ces temps: have + ed est une "périphrase du passé »; be + ing, une "périphrase du présent $»^{8}$. J'analyse cette dernière dans l'esprit indiqué.

\section{6}

\section{1}

$19 \mathrm{Au}$ commencement est une proposition imaginaire conjuguée au présent: John works. Dans mon exemple, celle-ci comprend l'idée d'un procès particulier identifié par le verbe et la référence à un individu susceptible d'y participer, ainsi qu'une conjugaison signifiant, à défaut d'indication contraire, la réalité du procès. On doit distinguer la proposition logique John - work, qui identifie le procès abstraitement, de la proposition conjuguée, qui peut avoir une contrepartie extralinguistique. La construction du sens étant progressive et cumulative, la simple idée du procès précède celle de l'actualisation. Quand la conjugaison intervient, celle-ci est dans le présent de l'énonciation et la proposition logique dans le passé de la construction. Ce fait aura son importance.

Figure 1

\begin{tabular}{|c|c|}
\hline $\begin{array}{c}\text { idée du procès } \\
\text { (proposition logique) }\end{array}$ & $\begin{array}{c}\text { actualisation représentée } \\
\text { (conjugaison) }\end{array}$ \\
\hline $\begin{array}{c}\text { passé de la } \\
\text { construction }\end{array}$ & $\begin{array}{c}\text { présent de } \\
\text { l'énonciation }\end{array}$ \\
\hline
\end{tabular}




\section{2}

20 Les constituants formels de la conjugaison sont un syntagme nominal parfois doté d'une marque spécifique, le « sujet ", et le temps, porté par un verbe. On admet que le sujet est thématisé et que le verbe à la tête du prédicat s'y rapporte. Le premier en est déterminé ; en retour, le second s'accorde en personne et en nombre. Indirectement, ces liens énonciatifs et syntaxiques expriment peut-être l'actualisation. Quand un procès s'actualise, le référent du sujet y participe effectivement. L'accord, signe que le verbe est lié, pourrait indiquer ainsi symboliquement participation et actualisation.

21 Peut-être la conjugaison dit-elle aussi l'actualisation par le temps grammatical. Entre autres fonctions, celui-ci verse le procès au temps, demeure de ce qui existe. Il le situe aussi par rapport à l'énonciation, cette précision constituant un autre indice d'existence.

\section{3}

22 La mise en rapport du procès avec l'« instant d'énonciation » constate une coïncidence, ou elle situe avant ou après le repère sur l'axe orienté du temps. Quand elle a joué, le procès est présent, passé ou futur, mais la nature du repère lui vaut des propriétés spécifiques en cas de coïncidence. Accueillant l'événement éphémère et singulier de l'énonciation, le présent est par convention un point, parfaitement identifié. Le procès dont l'actualisation coïncide avec lui, même partiellement, est localisé positivement, fixé intellectuellement au temps. Cette propriété est spécifique - les autres temps ne localisent pas le procès étroitement (v. infra) ; elle se perpétue, j'en fais l'hypothèse, dans les nominalisations en ing et explique certaines propriétés du participe présent et du gérondif. La langue par ailleurs est héritée et aucune énonciation n'est, tel le verbe johannique, un commencement : sur la ligne du temps le présent est médian. Il structure cette ligne doublement à partir de son centre en étant instant unique et instant parmi d'autres. Instant unique de l'énonciation, il s'oppose qualitativement au reste du temps et divise celui-ci en deux périodes différenciées - le passé (l'accompli, le mémoriel) et le futur (l'inaccompli, l'imaginé). Simple instant au milieu du temps, il prolonge l'instant précédent avant d'être prolongé à son tour et il contribue à la continuité et à l'homogénéité du substrat temporel. Séparateur et unificateur, il est le seuil où l'inaccompli du temps s'actualise et devient l'accompli. Le procès présent, installé sur une frontière et sur un continuum, est libre, s'il n'est pas ponctuel, d'aller au-delà du repère dans le passé et le futur, voire d'être coextensif à ces époques en couvrant l'entier du temps dans les "vérités générales ». Il se divise alors en une partie accomplie et une partie inaccomplie, le présent étant l'instant de son accomplissement et de son interception. Les valeurs aspectuelles traditionnelles du participe présent sont proches.

\section{7}

\section{1}

La proposition conjuguée est transformée. Elle perd sa conjugaison. La fonction sujet disparaît. Le référent du sujet reste mentionné, à un autre cas : John working/him working / *he working; souvent il cesse de l'être : working. Le temps disparaît aussi. Le verbe où il 
était porte désormais un suffixe ing (dans une autre genèse, il prend la marque du participe passé). Je fais l'hypothèse que cette perte n'est qu'une occultation et que la signification de la conjugaison perdure. Cette dernière se déployait dans le présent de l'énonciation (cf. supra). Elle se condense sous l'unique suffixe ing, et elle passe à l'arrièreplan, libérant le présent énonciatif pour une éventuelle nouvelle proposition, rectrice de ce fait. La proposition suffixée se destine ainsi à la subordination : à devenir une partie d'un autre constituant. Elle se "nominalise»: sans avoir la forme d'un syntagme nominal, elle en acquiert la fonction. Un tel syntagme, dans une proposition, est complément du verbe avec la fonction sujet, objet, attribut ou complément d'une préposition avec un rôle périphérique de complément d'attribution ou de circonstant; dans une phrase complexe, une proposition participiale ou gérondive a l'une ou l'autre de ces fonctions.

\section{2}

$24 \mathrm{Au}$ "passé », une identité morphologique fréquente souligne, en anglais moderne, l'enchaînement temps-participe; pourquoi rien de semblable ne s'observe-t-il au "présent»? Pourquoi walked seulement mais walk / walks et walking? À cela, deux grandes raisons ${ }^{9} .1^{\circ}$ ) Le présent est sans marque propre. Vraisemblablement, s est d'abord un indice de troisième personne du singulier. La base verbale, employée par tous les verbes sauf be, marque le présent seulement parce qu'elle est dans la conjugaison; elle indique l'infinitif ailleurs. Signifiant le procès, elle le rend présent à la conscience de l'énonciateur et elle convient quand l'actualisation coïncide, même partiellement, avec l'énonciation, mais elle ne contient, ni ne peut communiquer aux participes, aucune opération de localisation spécifique, si bien que le participe doit signifier son " présent » par ses propres moyens. Autre est la situation du passé. Si le procès est passé, une marque spécifique, ajoutée à la base verbale, doit le dire $(e d)$. La forme verbe + ed possède une valeur propre et une marque du passé ; cela facilite le transfert. $2^{\circ}$ ) Avec la conjugaison, les temps grammaticaux occupent le présent de l'énonciation tandis que les participes sont à l'arrière-plan énonciatif, dans le passé de l'énonciation (cf. supra). Une homologie entre la construction énonciative et le temps référentiel fait correspondre les temps grammaticaux et le présent, les participes et le passé.

Figure 2

\begin{tabular}{|l|c|c|}
\hline & $\begin{array}{c}\text { participes } \\
\text { (passé énonciatif) }\end{array}$ & $\begin{array}{c}\text { temps grammaticaux } \\
\text { (présent énonciatif) }\end{array}$ \\
\hline construction énonciative & $\longleftarrow$ passé & $\longleftarrow$ présent \\
\hline axe du temps & $\longleftarrow$ & $\longleftarrow$ \\
\hline
\end{tabular}

Disant le passé, le prétérit rend le procès homologue des participes; ainsi déterminé, celui-ci peut devenir l'un d'eux. Un procès au présent, en revanche, n'est jamais l'homologue des participes; aucune extension n'est possible. 


\section{1} présupposante. Dans les énoncés I can smell something burning ou I like driving this car, le verbe en ing réfère à un procès réalisé présupposé par celui de la principale. Dans l'hypothèse proposée, cette propriété provient du présent de l'indicatif sous-jacent, également actualisant. Deux précisions sont nécessaires cependant. $1^{\circ}$ ) La langue construisant des représentations, l'actualisation du présent et des nominalisations en ing est seulement représentée. Elle peut donc avoir une contrepartie extralinguistique (quelque chose brûle effectivement; je conduis cette voiture en ce moment ou habituellement). Ou être théorique: seeing is believing (when / if you see, you believe) ou fictive: She is afraid of falling into the water (she may fall into the water); There is no denying that... (one can't deny...). $2^{\circ}$ ) L'actualisation n'est pas représentée seulement par le verbe en ing. Elle l'est aussi par le participe passé de parfait (His father gone, ...) ou de passif (With John severely wounded, ...) ou par certains infinitifs (I saw him cross the street / He happened to be there / I rejoice to hear that...). Cette précision signifie que l'actualisation héritée du présent ne définit pas parfaitement la nominalisation en ing, même si, il est vrai, la coïncidence la rend saillante.

\subsection{1}

Dans la conjugaison, le prédicat (la notion du procès) détermine le sujet thématisé. Même si la nominalisation occulte ce dernier, elle conserve aussi la mémoire de ce rapport et le prédicat en ing a parfois une valeur adjectivale: They heard him talking on the phone, He died defending his country, John being late,..., Being late, .... La nature verbale et adjectivale de la forme ainsi employée explique la dénomination traditionnelle " participe $»^{10}$.

Il arrive que le participe s'adjective complètement: a charming girl. L'évolution est naturelle. Loin d'être une unité de signification primitive, un adjectif (ex. : beau) condense l'évocation d'une première réalité (la beauté) et un lien à une seconde référence indépendamment identifiée (homme) et déterminée. Cette partie du discours accueille donc les participes, dont la forme réunit aussi une notion lexicale et un lien préconstruit de détermination.

30

La qualité évoquée par l'adjectif, devenue propriété d'un référent, lui est associée en permanence ou tend à l'être. Quand le participe s'adjective, il désigne aussi un état. Le participe passé signifie l'accompli du procès; l'adjectif dérivé désigne l'état résultant: fallen rocks, vanished civilisations, grown fir-trees, a retired colonel, a locked and barred door ${ }^{11}$. Signifiant l'inaccompli, l'accomplissement en partie réalisé, en partie à venir, le participe présent favorise d'autres valeurs. Si le procès est atélique, il peut constituer directement une propriété permanente (Sleeping Beauty, bristling fir-trees); s'il n'est pas constamment actualisé, il se mue en catégorisation (the working man, falling rocks). Si le procès est télique, son actualisation ne peut être constante non plus. Le participe adjectivé inscrit en permanence dans le référent une disposition à actualiser, démontrée par les réalisations 
passées et en annonçant d'autres: surprising news, folding-chair, man-eating tiger. L’inaccompli conduit à la modalité.

\subsection{2}

31 L'hypothèse d'une continuité entre formes conjuguées et non conjuguées explique le sens des participes adjectivés mais aussi leur nombre. À l'indicatif la conjugaison de l'anglais oppose le présent et le prétérit, où le verbe lexical peut prendre le temps, et le futur, où ce dernier est porté par un auxiliaire d'origine modale. Cette différence reflète le fait qu'au passé et au présent, le procès, actualisé sauf indication contraire, peut être constaté simplement alors qu'un procès futur, même certain, est potentiel et dépend entièrement d'un point de vue. La périphrase du futur shall/will + infinitif détermine le sujet faiblement en lui apportant non le verbe lexical mais un auxiliaire. Sa nominalisation to + infinitif a une fonction adjectivale (She is the person to see / to see you) ou adverbiale (To hear you, people might think...) mais elle ne produit pas d'adjectif ${ }^{12}$. Le verbe lexical conjugué au présent ou au prétérit, au contraire, détermine le sujet fortement en lui apportant directement le procès actualisé. Après nominalisation, il est susceptible de désigner une propriété en devenant adjectif.

\subsection{1}

La forme en ing conserve du présent de l'indicatif l'actualisation du procès et la détermination du sujet. Du prétérit, le participe passé hérite des mêmes valeurs. Comme il a été suggéré en 5., la localisation temporelle sous-jacente explique les différences, aspectuelles et autres, entre ces deux nominalisations.

À l'instant $\mathrm{T}_{0}$ de l'énonciation la proposition reçoit un temps grammatical choisi selon le rapport du procès à ce même instant ; s'il coïncide partiellement ou totalement avec ce repère le procès est " présent " $(v \text {. supra })^{13}$. La proposition conjuguée se décale ensuite dans le préconstruit (v. supra). Sa coïncidence avec $\mathrm{T}_{0}$ cesse, mais la nominalisation en ing conserve ses effets, comme la localisation positive ou la situation médiane (cf. 6.3) : elle inscrit dans le procès une coïncidence abstraite compatible avec tous les repères. C'est une propriété des nominalisations de généraliser dans une forme et de rendre absolus des rapports originellement particuliers et éphémères.

L'intégration par la proposition de la coïncidence avec le repère présent a pour principal effet d'accroître son caractère nominal. Un nom a une incidence interne ${ }^{14}:$ l'ensemble de qualités liées qu'il condense ne se rapporte pas hors de lui à un référent identifié indépendamment dans le discours - ceci correspond à l'adjectif -, mais à un élément de sa signification : la partie spécifique de la notion " saumon » se rapporte à un " poisson », partie générique de cette même notion (« un saumon est un poisson qui... »). De même, dans les nominalisations jusqu'ici la relation du verbe au sujet, explicitée dans la conjugaison, est intégrée au premier, de sorte que celui-ci, trouvant en lui un support, possède une incidence interne et commence à se replier sur lui-même tel un nom. Or, au présent le repère temporel est aussi le site d'occurrence du procès. La conservation d'une coïncidence à un repère signifie que le verbe en ing a en lui également du temps auquel il échoit, un socle temporel. Cela accroît son incidence interne et accentue la nominalisation: la forme en ing constitue, plus que d'autres nominalisations, une référence autonome, séparée et stable ${ }^{15}$. 

plus nominale de "gérondif ». Dans ce cas, sa référence peut se lier, dans un nouveau procès, à d'autres réalités également autonomes : de nombreuses propositions gérondives sont les arguments d'un verbe et jouent un rôle de sujet ou d'objet dans une phrase complexe. Ou sa référence peut être un repère. Le gérondif figure à l'exception d'autres nominalisations dans les syntagmes prépositionnels exprimant divers repérages (On entering the room,...; This is achieved by V-ing...). Mieux que d'autres nominalisations il occupe la fonction sujet, représentant le thème installé auquel le prédicat se rapporte dans l'énoncé : It's fun to drive/? driving; Driving is fun/? To drive is fun. L'hypothèse du présent sous-jacent explique l'existence du gérondif et deux propriétés importantes de cette forme.

\subsection{2}

36

Incident à son sujet et à son site d'occurrence, le procès nominalisé détermine ceux-ci. Cette détermination intégrée mériterait une étude car elle est peut-être la principale raison de la préférence donnée en discours à la nominalisation en ing. Les propositions participiales circonstancielles illustrent la détermination du site d'occurrence quand elles contribuent à une relation causale. Dans l'énoncé Being late, I decided to catch a bus la participiale désigne un procès actualisé en indiquant qu'il a des implications pour la situation d'occurrence (Je compris que je n'arriverais pas à temps, que je devrais courir... ). C'est à ces implications que s'enchaîne I decided to catch a bus.

Cet élément de "pertinence présente», reconnu traditionnellement dans le fonctionnement du parfait, pourrait expliquer le choix, dans de nombreux contextes, de la périphrase be + ing - ou d'autres périphrases en ing. Ainsi le procès de You are forgetting your lighter est un trait situationnel imprévu qui doit, aux yeux de l'énonciateur, être pris en compte ici et maintenant et devrait susciter une réponse de l'allocutaire. L'énoncé Look! It's snowing! attire l'attention sur un événement qui modifie la situation. I must stand a minute or two here on the bridge though the clouds are threatening... est énoncé par un narrateur conscient du danger représenté par les nuages. I couldn't stand hearing the girl I loved insulted signifie que ce que j'entendais m'affectait au moment où je le percevais, etc.

Enfin la détermination du sujet et/ou de la situation d'occurrence explique vraisemblablement de nombreux gérondifs. Soit les énoncés Try pushing the car; She was afraid of waking up her mistress; He tried to avoid answering any question; They are unlikely to risk holding an election; Do you mind me smoking? Would you mind opening the window? Missing the train means waiting for an hour ; I don't want people coming in and talking all day; We are contemplating reforming the tax system. Dans chacun le procès au gérondif est actualisé en représentation. Dans chacun également, l'actualisation a des conséquences imaginées ou prévisibles, positives ou négatives: pousser fera peut-être démarrer la voiture ; la réveiller aurait sûrement courroucé la maîtresse de maison ; répondre l'aurait mis en difficulté; des élections peuvent être synonymes de défaite; si je fume, vous risquez d'être incommodé; si vous ouvrez la fenêtre, vous aurez moins chaud; si nous manquons le train, nous devrons attendre ; attendre lasse ; que les gens entrent et sortent continuellement m'irrite ; une réforme de l'impôt aura forcément des effets. Enfin dans la plupart des énoncés, le verbe dominant le gérondif exprime une réponse aux conséquences envisagées : puisque la voiture démarrera peut-être, tente l'expérience de la pousser ; réveiller la maîtresse de maison n'étant pas souhaitable, elle craignait de le 
faire ; répondre étant gênant, il chercha à l'éviter ; si une défaite est possible, organiser des élections comporte un risque ; si la fumée vous incommode, j'imagine que vous vous opposerez à ce que je fume ; des entrées et sorties continuelles m'irritant, je les refuse ; si réformer l'impôt doit avoir les conséquences positives que nous croyons, nous envisageons cette réforme. Chaque fois le procès subordonné précède en genèse le procès supérieur; le gérondif y condense la représentation d'une actualisation et de son incidence plausible dans une situation.

\subsection{3}

Autre propriété importante, la nominalisation en ing conserve sous une forme abstraite la détermination temporelle donnée par le présent. Le procès est localisé par un repère quelconque (v. supra). Coïncidant avec lui, il l'intègre et peut devenir repère lui-même. Cette propriété est en évidence dans les propositions participiales. Dans les énoncés Being late, he decided to catch a bus; Crossing the street, he saw the figure of a man on the roof, les procès au participe sont d'abord localisés en coïncidence avec un repère implicite $(=H e$ was late [then] / He crossed the street [at one point]). Leur localisation positive intégrée permet ensuite qu'ils localisent le procès principal. L'aspect inaccompli dont il va être question explique la nature de ce second repérage. Un repère s'étirant sur la ligne du temps a des chances de transcender le repéré ; un effet de cadre et d'inclusion s'ensuit, qui suscite les valeurs de continuité logique, simultanéité, etc. D'autres fois, la participiale est repérée par le procès de la principale. Ainsi, dans We heard him talking on the phone; He died defending his country; He went out slamming the door, on comprend: He talked on the phone then [when we heard him]; He defended his country then [when he died] (l'énoncé accepte aussi la première analyse); He slammed the door then [when he went out]. Localisé par le procès principal, le procès au participe ne saurait repérer ce dernier, mais il lui est incident et il a une fonction qualificative. Il peut qualifier un participant (talking on the phone), un participant et le procès principal (defending his country) ou le seul procès principal (slamming the door). À la façon d'un adverbe de manière.

Enfin, le présent localise le procès ou un de ses instants au milieu du temps. Dans la nominalisation, le repère est quelconque mais il est représenté aussi au milieu d'un laps de temps, solidaire d'autres instants qui constituent avec lui le substrat et le site potentiel du procès. Si le procès dure il s'étend donc sur le substrat de part et d'autre du repère. Au moment de la coïncidence avec ce dernier il est en accomplissement : en partie accompli, en partie inaccompli ; il est intercepté, saisi de l'intérieur: He went out smiling; Being late, he decided to catch a bus. Si le procès est ponctuel, son aspect n'est pas modifié ; que je dise He reached the landing and stopped ou Reaching the landing, he stopped; As he went out, he slammed the door ou He went out, slamming the door; reach et slam restent ponctuels et ont lieu une seule fois. Dans l'analyse du participe présent on a souvent attribué au procès une continuité qui est d'abord celle du substrat temporel. La construction d'un laps de temps permet de figurer les instants postérieurs au procès ponctuel où se réalisent ses conséquences : le personnage est sur le palier, les témoins sont impressionnés par cette sortie brutale. Dans le premier exemple, ce substrat est le cadre du procès principal (v. supra). 


\section{4}

41 La localisation du prétérit est différente de celle du présent et cela limite la nominalisation issue de ce temps. Le repérage des procès décide d'abord s'il y a ou non une coïncidence, totale ou partielle, avec $\mathrm{T}_{0}$. Si c'est le cas le procès trouve immédiatement son site d'occurrence et une localisation positive (v. supra) ; comme une opération suffit au repérage le présent est simple morphologiquement. S'il n'y a pas coïncidence le repérage continue ; la morphologie plus complexe des autres temps traduit le supplément d'opération. $\mathrm{T}_{0}$ restant le repère, il s'agit d'indiquer à cette étape si le procès précède ou suit ce point sur l'axe orienté du temps. Là commence la différence. Alors que le présent est essentiellement un point, le passé et le futur, bornés à un bout par le présent et ouverts à l'autre sont de grands domaines. Un procès coïncidant avec le présent est localisé précisément et a des chances de dépasser son repère (v. supra). Un procès versé simplement au passé ou au futur par un temps grammatical de base a des chances, au contraire, d'en occuper une infime partie et, le site d'occurrence avec lequel il coïncide n'étant pas identifié, d'être sans localisation précise. Cette indétermination convient aux références à l'avenir car les procès potentiels n'ont pas encore rencontré leur site. En revanche elle fait difficulté au passé, qui est du temps réalisé, et le contexte se charge généralement de situer le procès, grâce à divers circonstants en particulier.

Si une conjugaison au prétérit sous-tend le participe passé, le procès désigné par celui-ci a été lié au référent du sujet - l'adjectivation est donc possible; il a été situé avant le repère - une visée rétrospective le saisit accompli ${ }^{16}$; mais, le prétérit ne l'ayant fait coïncider avec rien de précis, il n'a pas intégré l'instant auquel il est échu et il est sans socle temporel. À la différence des procès nominalisés par ing, il ne constitue donc pas une référence stabilisée et séparée pouvant être liée par un nouveau procès à d'autres référents autonomes. Aucun gérondif, autrement dit, n'est issu du prétérit ; on ne dit pas *I remember posted the letter, comme on le ferait peut-être si seul comptait le rapport au repère mais I remember posting the letter, avec une forme qui dit l'autonomie du référent. De même, n'ayant intégré aucun instant particulier le procès au participe passé ne repère rien. Vraisemblablement dans les participiales comme The book written,...His father gone, le participe passé est dominé par un participe présent en ellipse qui assure le repérage : The book being/having been written, His father having/being gone.

L'infinitif contribue à l'expression du futur. Il ne suppose pas un site d'occurrence identifié et sous sa forme nue le procès n'est pas une réalité stabilisée non plus. Comme il recouvre un lien théorique au sujet, il est moins qualitatif que le participe passé. En première analyse, to lui ouvre les mêmes fonctions nominales que le gérondif. Mais l'équivalence n'est pas totale. To montre une visée prospective et une afférence pendant lesquelles le procès, devenu un point cible, projeté, approché puis atteint, est implicitement fixé dans le temps. Cette fixation explique l'équivalence. Mais elle est donnée de l'extérieur par l'opération de to, elle n'émane pas de l'intégration par le procès d'une coïncidence à un repère temporel identifié et elle est plus faible que celle du gérondif. Cela se traduit par des restrictions syntaxiques. Outre qu'il n'a pas de rôle dans le repérage temporel des procès, alors que le gérondif est un repère après une préposition, cet infinitif est plus rarement sujet, car cette fonction thématise, et stabilise, le référent. 


\section{1} liés pour la première fois est un rapprochement, dont l'aboutissement spontané est cette valeur. Indiquant ce qui est en puissance dans toute relation sauf indication contraire certaines langues omettent parfois de marquer «être» entre des références liées syntaxiquement - be communique une information quasi nulle. Quand il est conjugué, le sujet reçoit principalement l'apport du procès complément. De ce fait la périphrase est comme une seconde conjugaison de la relation prédicative primitive. Elle contient trois strates. La proposition nominalisée représente la conjugaison ancienne; be la domine et figure une conjugaison potentielle; la conjugaison effective est au premier plan énonciatif. Si be rapporte le prédicat au sujet, il lui confère une valeur de détermination et la proposition en ing est participiale, comme le confirme l'impossibilité de la remplacer par it (He is coming $\rightarrow{ }^{*} \mathrm{He}$ is it).

Figure 3

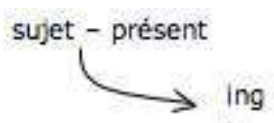

II

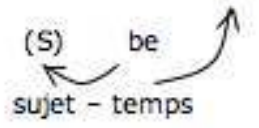

47

Cette analyse est l'occasion de préciser que la conjugaison rapporte au sujet la relation établie par be et que la structure contient une hiérarchie notionnelle dominée par ce 
verbe et une seconde, énonciative, dominée par le sujet, dont le référent est à la fois participant du procès et thème de l'énoncé. Les deux hiérarchies se succèdent. Le verbe lie les références tout en orientant la relation vers le sujet. Celui-ci conserve la relation dans son domaine. Revenons au sens de be.

\section{2}

Be n'est pas le seul auxiliaire rencontré dans les périphrases; celle du parfait emploie généralement have. Lexical, ce verbe dit la possession ou une variante. Il exprime l'inclusion dans le domaine d'un premier référent repère d'un second, distinct ou présenté tel, et il souligne la pertinence de cette inclusion pour le repère (Cotte, 1998). Auxiliaire, il indique vraisemblablement la relation temporelle construite par le prétérit sous le participe passé tout en disant la pertinence pour l'instant repère. Le passé contient en effet la même association-dissociation que la possession. Le temps est un axe structuré par l'instant repère du présent et il en constitue le domaine ; un procès passé est donc dans le domaine du présent sans coïncider cependant avec ce dernier. Cette analyse suggère que la coïncidence de be ne sert pas seulement à reconjuguer facilement le prédicat nominalisé, mais qu'elle figure la coïncidence du présent préconstruit. Be indique que le procès et le repère sont contemporains et que le premier détermine le second en lui étant incident ; ainsi il répète abstraitement mais fidèlement la conjugaison primitive occultée.

La structure be + ing est ainsi le produit d'une « réélaboration ». Ses strates contiennent une même signification qui gagne en explicitation et elle constitue une expansion du présent primitif. En généralisant le propos, l'analyse signifie que les constructions s'enchaînent sans solution de continuité à l'intérieur d'un «système génétique » où les plus anciennes contiennent en germe les nouvelles, qui gardent la mémoire de leur filiation. On passe d'une étape de la genèse à la suivante par une synthèse : la conjugaison explicite du présent se condense en une forme verbale unique en ing, ou par une analyse : be explicite la coïncidence. Les périphrases verbales ne sont pas analytiques sans qu'un constituant y synthétise une étape antérieure du sens : be + ing est une construction globalement analytique dont la partie subordonnée récapitule une conjugaison au présent et regarde vers le passé de l'énonciation.

50 La continuité entre la construction primitive et celle qui la prolonge n'implique pas une synonymie parfaite, car le prix de l'explicitation est l'abstraction. Be est spécialisé dans la signification de coïncidence et l'exprime indépendamment d'un repérage véritable par rapport à $\mathrm{T}_{0}$. Une coïncidence effective doit être signifiée par un dernier présent grammatical (be coming $\rightarrow$ he is coming) mais la nouvelle construction est disponible pour d'autres valeurs et elle peut s'employer avec d'autres temps.

51 Créant une construction sur fond d'identité, la langue se « renouvelle » aux deux sens du terme : elle explicite ses principes et elle se perpétue; ce faisant, elle crée une nouvelle structure et elle change. De la fidélité à l'ancien naît le neuf. On retrouvera en 9.4. la spécificité sémantique de la périphrase. 


\section{3}

52 La périphrase analyse le temps et met au jour ses opérations. La suivre dans son travail d'explicitation montre deux lois du langage : il y a de l'implicite dans la grammaire ; une « métalangue naturelle » existe.

Toute référence est construite ici et maintenant par un énonciateur ; son rapport à la situation d'énonciation est généralement indiqué par une marque grammaticale: les temps localisent en fonction de $\mathrm{T}_{0}$. Or, ce rapport, comme la coïncidence ou l'antériorité, est dit implicitement pour partie. Les temps du présent et du prétérit, bien différenciés morphologiquement, expriment clairement les valeurs rappelées. Cependant ils ne le font pas explicitement, comme coïncidence ou antériorité ou comme les verbes be et have, qui désignent des états manifestant ces valeurs. L'expression occupe la fin du lexème verbal ; elle se résume à une désinence et, pour la coïncidence, à un morphème zéro à plusieurs personnes. C'est peut-être la plus grande caractéristique de la grammaire d'offrir au sens une expression minimale. Celle-ci se traduit par une saillance moindre: dans la signification du mot la composante lexicale est en lumière; la valeur exprimée grammaticalement, marginalisée, en est une simple détermination.

Il y a plus. Le degré de l'explicitation traduit le rapport de l'énonciateur à la signification. S’il explicite celle-ci en lui consacrant, typiquement, un mot, l'énonciateur lui donne de l'autonomie ; il reconnaît qu'elle constitue un principe sémantique distinct, indépendant de ses occurrences et en particulier de l'énonciation où elle est dite. S'il ne l'explicite pas, il ne lui offre ni reconnaissance ni autonomie. La signification reste liée à l'énonciation qui la construit. Elle paraît sans généralité, strictement contemporaine de l'acte énonciatif qui la porte, peut-être mimée : par principe la conjugaison occupe le présent de l'énonciation et décale la représentation du procès dans le préconstruit (cf. 6.1.) ; un morphème de temps positif, comme celui du prétérit régulier, physifie la conjugaison, souligne l'écart génétique avec le procès et repousse celui-ci de façon affirmée dans le passé de la construction - manière indirecte d'exprimer le décalage chronologique du passé; le morphème zéro du présent a l'effet inverse: le procès n'est pas décalé formellement du présent génétique, ce qui exprime indirectement la coïncidence, l'immédiateté à l'énonciation. La signification devient vivante. Peut-être les repérages, pour rattacher véritablement à l'énonciation, doivent-ils participer concrètement de celle-ci, faire corps avec l'énonciateur.

La périphrase prend pour objet le présent, l'analyse et explicite une partie du sens. Comme H. Adamczewski à propos d'autres faits de langue, on peut parler de « métalangue naturelle ». Cette métalangue n'est pas celle dont un énonciateur décide afin d'identifier la prononciation, la forme, la fonction, la construction ou le sens d'un signe « autonyme ». Elle exprime dans la grammaire la réflexivité de la pensée, la capacité de l'esprit à étudier ses productions et à tirer le particulier vers le général. Elle témoigne pour la paraphrase, la synonymie, la traduction des signes, qui est peut-être la condition de leur existence.

\subsection{1}

Deux problèmes restent: $1^{\circ}$ ) Be se conjugue avec un sujet identifiant souvent un participant du procès, mais il signifie la coïncidence du procès entier avec la situation repère d'actualisation. Comment justifier cette rupture avec la lettre de la structure ? $2^{\circ}$ ) Be présente la coïncidence comme un état ; pourquoi ? 

Alors que le be lexical attribue le prédicat au sujet (ex.: He is rich/late / a liar/a good performer, etc.), l'auxiliaire paraît attribuer le procès à un site plus grand, qui peut être la situation repère (ex.: It is raining). Le référent du sujet est également concerné par l'attribution, dans la mesure où il appartient à cette situation. Dans He is watching television / lying / coming tomorrow ce référent n'est pas simplement $\mathrm{He}$ mais $\mathrm{He}$ - here - and - now, la personne localisée dans la situation repère étroite, définie éventuellement par le seul $\mathrm{T}_{0}(\mathrm{He}-$ now) ; glose : "J'attribue à $\mathrm{He}$ - (here - and -) now le procès watch television, etc ». L'analyse est généralisable. $1^{\circ}$ ) Avec certains verbes lexicaux, il est indispensable de distinguer l'incidence du prédicat dans la conjugaison et l'incidence du procès. Dans He happened to be here ou He is believed to be innocent, happen et believe ne concernent pas le seul sujet mais tout le procès subordonné. $2^{\circ}$ ) La périphrase du parfait et les périphrases à auxiliaires de modalité ont un fonctionnement semblable. Dans He has done it! l'état résultant est pertinent dans toute la situation repère (here and now) et le référent du sujet est également déterminé dans la mesure où il appartient à celle-ci (he here - and - now) : il doit être en vie au moment où l'on réfère au passé. Dans beaucoup d'emplois les auxiliaires modaux, de même, lient le procès subordonné tout entier à une instance extérieure (ex. : He may have done it). L'analyse, si elle est correcte, confirme la tendance à l'abstraction des verbes auxiliaires.

\section{4 .2}

La coïncidence est exprimée comme un état pour une raison structurelle. Dans le préconstruit de la périphrase le présent simple, fonctionnant sur le mode de l'implicite, opère la coïncidence dans l'instant étroit de l'énonciation (v. supra). Be n'effectue aucune coïncidence mais en explicite le principe. L'ayant extraite du préconstruit, il la porte à la dimension d'un mot, en fait un principe général autonome (v. supra), la dit en elle-même. Cela suppose sa stabilité et impose un verbe d'état: be est appelé par la démarche explicitante de la périphrase.

59

La périphrase signifie que le procès subordonné est dans un état de coïncidence; cela a des conséquences sur l'aspect. La coïncidence itérée aux différents instants de l'état est une concomitance; le procès repéré de multiples fois est dynamique: alors qu'une proposition participiale ne suppose pas l'itération d'un procès ponctuel (v. supra, He went out slamming the door), la périphrase la suggère (He was slamming the door, winking at me, etc). Sans être nécessairement recherchés pour eux-mêmes, les effets aspectuels de progressif, visée sécante intérieure, etc. sont produits mécaniquement par la forme, tout comme sont renforcés les effets déictiques. Un état dit une relation installée, indépendante de l'énonciation en cours ; le procès, préexistant à la référence, est un objet du monde vu en situation; il est montré et l'ostension peut être continuée ; be + ing est proche de this. D'autres effets plus abstraits suivent : l'ostension continuée saisit le procès réel derrière les apparences, l'identifie, l'analyse, signale qu'il interprète un premier procès, souligne sa pertinence pour la situation repère, etc. (une description de ces valeurs est donnée dans Cotte 1997, 96-116). Pour ne rien dire du sujet. Les formes en ing et be + ing signifient-elles le contrôle du procès par celui-ci (cf. He is being silly, He began studying law)? Ou une incidence marquée, quel que soit le rôle actanciel (cf. The book is printing)? Ces questions nous attendent. 


\section{4 .3} aspectuel, modal ou textuel, présent dans chaque occurrence, qui expliquerait l'emploi de la forme. Peut-être cet invariant existe-t-il. Le meilleur candidat actuellement semble être "la pertinence présente", l'incidence au site d'occurrence, avec sa traîne de réactions modales, interprétatives, etc. Cependant, l'analyse montre qu'une telle valeur, si elle se laisse observer dans chaque occurrence de la forme, est solidaire de nombreuses autres, en particulier des valeurs aspectuelles traditionnelles, qui sont générées par la structure et, à ce titre, pertinentes. Il n'y a pas un invariant, seul pertinent, et des effets contingents négligeables, mais un invariant et des effets caractéristiques, sollicités par certains contextes (à identifier). La périphrase doit être définie par l'invariant mais aussi par ce qu'elle peut seule signifier. Son identité est moins une valeur unique que l'unité sémantique des significations qui lui sont associées.

Longtemps on a aimé exclure; je propose d'intégrer ; pour être fidèle à la langue et, comme elle, créer en reconnaissant.

\section{BIBLIOGRAPHIE}

Adamczewski, H. 1982. Grammaire linguistique de l'anglais. Paris : A. Colin.

Auroux, S. (dir.). 1990. Les notions philosophiques, dictionnaire. Paris : Presses Universitaires de France.

Bach, E. 1967. « Have and be in English syntax ». Language 43, 462-485.

Cotte, P. 1996. L'explication grammaticale de textes anglais. Paris : Presses Universitaires de France.

Cotte, P. 1997. Grammaire linguistique. Paris : Didier-Érudition.

Cotte, P. 1998. « Have n'est pas un verbe d'action : l'hypothèse de la réélaboration ». In A.

Rousseau (dir.), La transitivité. Presses Universitaires du Septentrion, 415-439.

Guillaume, G. 1964. Langage et science du langage. Paris : Libraire A-G. Nizet ; Québec : Les Presses de l'Université Laval.

Guillaume, G. 1965 [1929]. Temps et verbe. Paris : Librairie ancienne H. Champion.

Guillaume, G. 1973. Leçon de Linguistique 1948-49, série C, volume 3, « Grammaire particulière du français et grammaire générale (IV) », Paris : Klincksieck : Québec : Les Presses de l'Université Laval.

Guillaume, G. 1982. Leçons de Linguistique 1956-57, volume 5, « Systèmes linguistiques et successivité historique des systèmes II ». Lille : Presses Universitaires de Lille ; Québec : Les Presses de l'Université Laval.

Guillaume, G. 1992. Leçons de Linguistique 1943-44, série A, volume 10, « Esquisse d'une grammaire descriptive de la langue française (II)». Lille : Presses Universitaires de Lille ; Québec : Les Presses de l'Université Laval. 
Guillaume, G. 1993. Leçons de Linguistique 1938-39, volume 12, Lille : Presses Universitaires de Lille ; Québec : Les Presses de l'Université Laval.

Houdé, O. et al. 1998. Vocabulaire des sciences cognitives. Paris : Presses Universitaires de France. Jespersen, O. 1909-49. A Modern English Grammar on Historical Principles I-VII. Copenhague : Munksgaard.

Joly, A. et D. O'Kelly. 1990. Grammaire systématique de l'anglais. Paris : Nathan.

Le Goffic, P. (dir.). 1986. Points de vue sur l'imparfait. Caen : Presses Universitaires de Caen.

\section{NOTES}

1. Voir J. Deschamps, article « genre » in Auroux éd. (1990).

2. Voir les articles « héritage » et « réseau sémantique » dans Houdé et al. (1998).

3. Sur le temps opératif, la notion de système diachronique et l'idéogénèse, cf. Guillaume 1939 : 109 ; 1943-44 : 138-9, 141, 143, 226, 243 ; 1956-7 : 164 ; 1964 : 84.

4. L'infinitif n'a ni personne ni temps explicites; le subjonctif introduit la personne, mais ne rapporte pas le procès, pour le localiser, au présent de l'énonciation; l'indicatif s'enrichit de ce repérage ; cf. G. Guillaume (1929).

5. Voir G. Guillaume 1964 : 143-183. L'interprétation du défini générique m'est propre.

6. La triade culiolienne " parcours - extraction - fléchage » leur est redevable. A. Joly est, chez les anglicistes, le plus fidèle continuateur (Joly \& O'Kelly 1990).

7. Sur l'imparfait français, cf. récemment Le Goffic (1986).

8. L'idée n'est pas neuve. E. Bach (1967) met un « embedded past » dans le parfait.

9. Sur ce point, voir P. Cotte (1996: 74-75).

10. Un procès détermine aussi la situation où il a lieu. La proposition participiale peut donc avoir une fonction adverbiale Being late, he decided to catch a bus.

11. Sur l'adjectivation de ces participes, voir P. Cotte $1996: 83-92$.

12. Un participe futur existe en latin pour les procès proches et certains (scripturus sum : je vais écrire) et il se nominalise (morituri te salutant). On peut donc caractériser, voire identifier, un référent par un procès futur, mais l'opération est vraisemblablement plus délicate. Le français est sans participe futur bien que le futur y soit morphologique; l'anglais souligne le potentiel du futur et a encore moins de raisons de construire une telle forme.

13. La coïncidence est totale quand le procès présent est ponctuel ; elle est partielle sinon. Elle est rapprochée quand le procès est « en situation »; elle l'est moins quand le rapport est entre la situation repère et le laps de validité d'un procès éternel ou cyclique traité comme une propriété (The sun sets in the West).

14. Le concept d'incidence interne est dû à G. Guillaume (1973:61).

15. L'incidence interne connaît des degrés. Dans le nom verbal (ex. the building of the cathedral), le verbe indique son incidence à l'objet et accroît son caractère nominal (Cotte $1986: 253$ sq).

16. Le participe passé du passif indique l'accompli de l'actif (Cotte 1996 : 80). 


\section{RÉSUMÉS}

L'étude présente une nouvelle théorie de fonctionnement interne de la périphrase be-ing en anglais contemporain. Elle développe l'intuition que cette structure réélabore une conjugaison sous-jacente au présent en répétant des éléments de sa signification dans le participe et dans le verbe vecteur be. Elle tente de dériver les propriétés aspectuelles et autres de la forme du processus de la réélaboration.

This article propounds a new theory of the internal organisation of the be-ing periphrasis in contemporary English. It is based on the idea that the structure "re-elaborates" an underlying finite verb in the present tense, reiterating elements of meaning in the participle and superordinate verb be. It is shown that aspectual and other semantic properties may ultimately be derivable from the process of re-elaboration.

\section{INDEX}

Mots-clés : aspect, be-ing, forme périphrastique, gérondif, grammaire métaopérationnelle, participe présent, psychosystématique, réélaboration, sens explicite, sens implicite, temps Keywords : aspect, be-ing, gerund, meaning (explicit), meaning (implicit), metaoperational grammar, periphrastic form, present participle, psychosystematic, re-elaboration, tense

\section{AUTEUR}

\section{PIERRE COTTE}

Pierre Cotte est professeur de linguistique anglaise à l'Université de Paris-Sorbonne, après avoir enseigné aux universités de Brest et de Lille. Il a publié L'explication grammaticale de textes anglais (1996 Presses Universitaires de France), Grammaire linguistique (1997 Didier) ainsi qu'une trentaine d'articles de grammaire anglaise depuis 1980. Il est membre de l'UFR SILEX et il a été président de l'ALAES de 1993 à 1999.pierre.cotte@paris-sorbonne.fr 